\title{
Voice-supported Electronic Health Record for Temporomandibular Joint Disorders
}

\author{
R. Hippmann ${ }^{1,2}$, T. Dostálová, ${ }^{1,2}$, J. Zvárová, ${ }^{3,2}$, M. Nagy $^{3,2}$, M. Seydlova $^{1,2}$, P. Hanzlícek ${ }^{3,2}$, P. Kriz', I. Smidl', J. Trmal ${ }^{4}$ \\ ${ }^{1}$ Department of Paediatric Stomatology, Second Faculty of Medicine of Charles University, Prague, Czech Republic \\ ${ }^{2}$ Centre of Biomedical Informatics, Prague, Czech Republic \\ ${ }^{3}$ EuroMISE Centre, Department of Medical Informatics, Institute of Computer Science, v.v.i., Academy of Sciences of the Czech Republic, \\ Prague, Czech Republic \\ ${ }^{4}$ Department of Cybernetics, University of West Bohemia, Plzen, Czech Republic
}

\begin{abstract}
Summary
Objective

Biomedical ontologies exist to serve integration of clinical and experimental data, and it is critical to their success that they be put to widespread use in the annotation of data. How, then, can ontologies achieve the sort of userfriendliness, reliability, cost-effectiveness, and breadth of coverage that is necessary to ensure extensive usage?

\section{Methods}

Our focus here is on two different sets of answers to these questions that have been proposed, on the one hand in medicine, by the SNOMED CT community, and on the other hand in biology, by the OBO Foundry. We address more specifically the issue as to how adherence to certain development principles can advance the usability and effectiveness of an ontology or terminology resource, for example by allowing more accurate maintenance, more reliable application, and more efficient interoperation with other ontologies and information resources.
\end{abstract}

\section{Results}

SNOMED CT and the OBO Foundry differ considerably in their general approach. Nevertheless, a general trend towards more formal rigor and cross-domain interoperability can be seen in both and we argue that this trend should be accepted by all similar initiatives in the future.

\section{Conclusions}

Future efforts in ontology development have to address the need for harmonization and integration of ontologies across disciplinary borders, and for this, coherent formalization of ontologies is a pre-requisite.

\author{
Keywords \\ Quality Assurance, SNOMED CT, \\ Biomedical ontologies, ontology harmo- \\ nization
}

DOI

10.3414/ME9302

The full English version can be found in /Methods of Information in Medicine 2010 49 2: $168-172 /$ or http://www.schattauer.de/de/magazine/ue bersicht/zeitschriften-az/methods/issue/special/manuscript/1231 0/show.html. 